\title{
EMI CONDUZIDA GERADA POR CONVERSORES ESTÁTICOS APLICADOS À CORREÇÃO DO FATOR DE POTÊNCIA
}

\author{
Fernando Beltrame* \\ engbeltrame@gmail.com \\ Luciano Schuch $^{\dagger}$ \\ schuch.prof@gmail.com
}

\author{
Leandro Roggia* \\ roggia@gmail.com \\ José Renes Pinheiro ${ }^{\dagger}$ \\ renes@ctlab.ufsm.br
}

*Instituto Federal de Educação, Ciência e Tecnologia do Rio Grande do Sul - IFRS

Rua Nelsi Ribas Fritsch, 1111

CEP 98200-000, Ibirubá - RS, Brasil

${ }^{\dagger}$ Universidade Federal de Santa Maria - UFSM, Grupo de Eletrônica de Potência e Controle - GEPOC

Av. Roraima, 1000, prédio 10

CEP 97105-900, Santa Maria, RS - Brasil

\begin{abstract}
Conducted EMI Yield by Static Converters Applied to Power Factor Correction

This paper investigates the conducted electromagnetic interference (EMI) yielded by power converters used power factor correction. The topologies discussed in this paper are the boost, interleaved boost and dual boost converters. An analysis of the conducted noise behavior of differential mode and common mode in the converters are presented. All converters are designed in a way that the total harmonic distortion of the input current are same, in order to use the same input filter. It is presented the EMI filter design in order to adapt the converters as the standard of electromagnetic interference, CISPR. Experimental results of converters operating in power factor correction for a load of $300 \mathrm{~W}$ is presented, as well as, experimental results of EMI analyzing the impact of the use of EMI filter, the alteration of the gate resistance of the semiconductor switches and changed in the configuration of the inductor boost for dual boost topology.
\end{abstract}

Artigo submetido em 10/12/2010 (Id.: 01231)

Revisado em 10/02/2011, 11/04/2011

Aceito sob recomendação do Editor Associado Prof. Francisco de Assis dos Santos Neves
KEYWORDS: Boost, interleaved boost, dual boost, EMI filter, PFC, DM and CM noise.

\section{RESUMO}

Este trabalho investiga a interferência eletromagnética (EMI) conduzida gerada por conversores estáticos aplicados a correção do fator de potência. As topologias discutidas neste artigo são os conversores boost, boost intercalado e dual boost. Uma análise do comportamento dos ruídos conduzidos de modo diferencial e de modo comum nos conversores são apresentados. Os conversores são projetados de forma que a taxa de distorção harmônica da corrente de entrada seja a mesma, com o objetivo de usar o mesmo filtro de EMI de entrada. É apresentado o projeto do filtro de EMI com o objetivo de adequar os conversores a norma de interferencia eletromagnética (CISPR 22). Resultados experimentais dos conversores operando na correçao do fator de potência para uma carga de 300W são apresentados, bem como, resultados experimentais de EMI analisando-se o impacto da utilização do filtro de EMI, da alteração da resistência de gate das chaves semicondutoras e alteração da configuração do indutor boost na topologia dual boost. 
PALAVRAS-CHAVE: Boost, boost intercalado, dual boost, filtro de EMI, PFC, ruído DM e CM.

\section{INTRODUÇÃO}

O emprego do conversor boost operando na correção do fator de potência (PFC), com o intuito de adequação com as normas referentes aos harmônicos de baixa ordem, está se tornando cada vez mais comum em várias aplicações da indústria. Esta aplicação, no entanto, soluciona os problemas quanto aos harmônicos de baixa ordem. Por outro lado, gera harmônicos de alta ordem, EMI conduzida. Tais harmônicos são gerados, principalmente, devido aos processos de comutação empregados nos conversores aplicados como PFC, como apresentado por (Yang et al., 2004) e (Lu et al., 2004). Associado aos processos de comutação, têm-se as altas taxas de variação de corrente $(d i / d t)$ e as altas taxas de variação de tensão $(d v / d t)$, as quais, são os reais responsáveis pela a geração de EMI conduzida em conversores comutados.

No caso das altas taxas de variação de corrente (em conversores comutados são representadas pelas correntes pulsadas) a geração de EMI está associada a corrente de entrada dos conversores que estão comutando em alta frequência, gerando harmônicos múltiplos da freqüência de comutação, dentro dos limites de EMI conduzida (CISPR 22). Esses harmônicos de corrente de alta frequência são conhecidos como ruído conduzido de modo diferencial (DM), como mostrado por (Zientarski et al., 2009) e (Mainali and Oruganti, 2010).

Por outro lado, quanto os harmônicos gerados pelas altas taxas de variação de tensão, os mesmos dependem da variação da amplitude da tensão, da frequência e dos tempos de comutação. Estas variações de tensão se transformam em correntes de ruído conduzido através da carga e descarga das capacitâncias parasitas presentes nos conversores, resultando em correntes de alta frequência, as quais circularão para rede. Tal tipo de ruído, o qual está relacionado com os altos $d v / d t$, é conhecido como ruído conduzido de modo comum (CM), conforme (Zientarski et al., 2009) e (Mainali and Oruganti, 2010).

Portanto, devido à grande geração de EMI conduzida por conversores comutados, este trabalho apresenta uma investigação do ruído gerado pelos principais conversores aplicados como PFC. Os conversores sob análise são o conversor boost, ilustrado na Figura 1, o conversor boost intercalado, ver Figura 2 e o conversor dual boost, apresentado na Figura 3. Uma análise dos ruídos conduzidos do tipo DM e CM são apresentados, bem como uma ilustração da circulação destes ruídos nos conversores. O projeto do filtro de EMI utilizado é apresentado, bem como resultados experimentais da EMI gerada por estes conversores com e sem a utilização do filtro de EMI, com alteração da resistência do gate das chaves semicondutoras e com alteração do indutor boost para a topologia dual boost. Resultados experimentais dos conversores operando como PFC para uma carga de 300 W são apresentados.

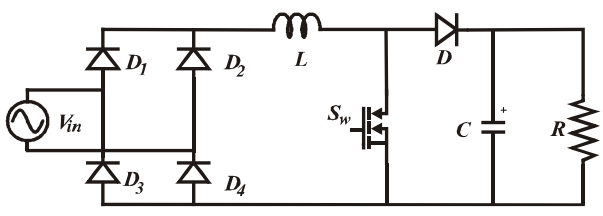

Figura 1: Conversor boost.

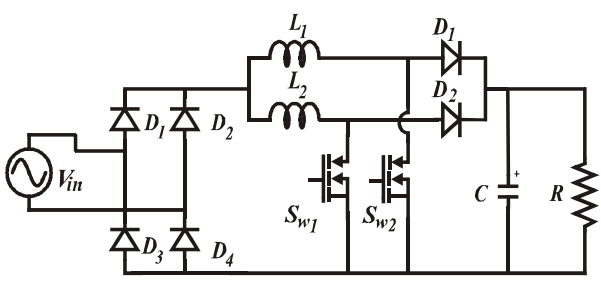

Figura 2: Conversor boost intercalado.

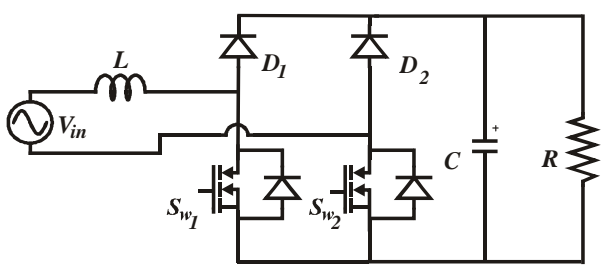

Figura 3: Conversor dual boost.

\section{EMI CONDUZIDA}

Em conversores comutados a EMI conduzida se apresenta de duas formas distintas, através do ruído conduzido DM e ruído conduzido CM. A corrente de ruído DM, ilustrado na Figura 4, ou também chamado de ruído simétrico, circula sobreposta a própria corrente de alimentação do conversor, com a única diferença de ter frequências superiores. Tal tipo de ruído é gerado principalmente por corrente pulsantes, comutações de turn-on e turn-off dos dispositivos semicondutores e pelas oscilações causadas pela corrente de recuperação reversa dos diodos, de acordo com (Rossetto et al., 2000).

Por outro lado, o ruído CM, ou também chamado de ruído assimétrico, circula pelo condutor de aterramento ver Figura 5, e utiliza como caminho os elementos parasitas existentes entre o circuito e o chassi do equipamento, que por questões de segurança é aterrado. Nesse caso, os grandes responsáveis pelo aparecimento de ruído conduzido $\mathrm{CM}$ em conversores comutados são os parasitas capacitivos e as altas variações de 
tensão ( $d v / d t)$, conforme (Yang et al., 2004), (Lu et al., 2004) e (Lissner et al., 2007).

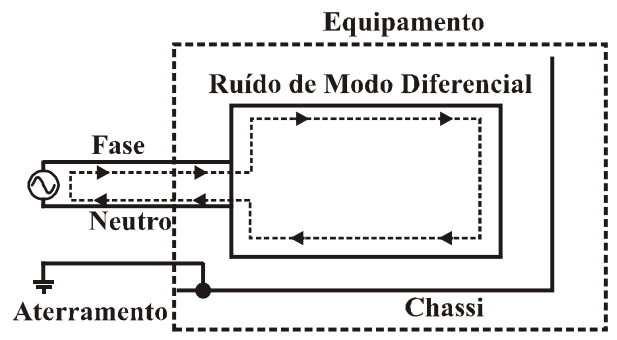

Figura 4: Circulação do ruído conduzido DM em um equipamento qualquer.

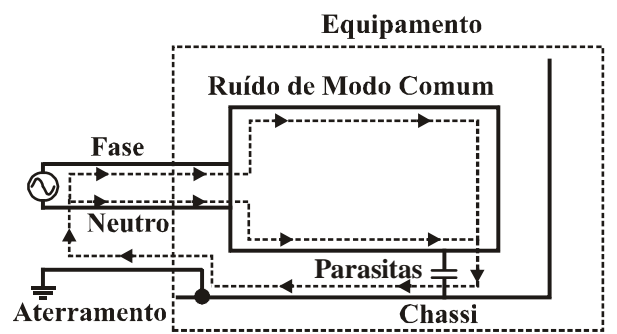

Figura 5: Circulação do ruído conduzido $\mathrm{CM}$ em um equipamento qualquer.

\subsection{EMI Conduzida - Modo Diferencial}

O ruído conduzido do tipo DM tem uma ligação direta com as altas de variações de corrente. Logo, é correto afirmar que este tipo de ruído tem forte dependência na forma de onda da corrente de entrada. Devido ao fato da etapa PFC ser a responsável pela definição da ondulação e da freqüência da corrente de entrada (através do projeto do indutor boost), pode- se dizer que, esta etapa é a principal responsável pela geração de ruído conduzido DM que será injetado na rede elétrica, conforme afirma (Pieniz et al., 2006) e (Zientarski et al., 2009). Tal ruído circula sobreposto à própria corrente de alimentação do equipamento. Logo, o caminho percorrido pelo ruído DM é o mesmo percorrido pela corrente principal do conversor.

Em conversores operando como PFC, a forma de onda da corrente de entrada, tem como característica a forma de uma onda triangular, a qual gera EMI conduzida com frequências maiores que a frequência de comutação. Através da transformada de Fourier desta forma de onda triangular é obtida a equação (1), como demonstrado por (Lu et al., 2004).

$$
i(t)=\frac{8 I_{1}}{\pi^{2}} \sum_{n=1,3,5, \ldots}^{\infty} \frac{(-1)^{\frac{(n-1)}{2}}}{n^{2}} \sin \left(\frac{2 n \pi}{T} t\right)
$$

Onde:

$$
\begin{aligned}
& i(t) \text { - Corrente senoidal. } \\
& I_{1} \text { - Componente fundamental da corrente. } \\
& T \text { - Período. } \\
& n \text { - Ordem do Harmônico. }
\end{aligned}
$$

A partir da equação (1), pode-se concluir algumas das propriedades do ruído conduzido DM:

- A frequência do ruído é múltiplos inteiros da frequência de comutação.

- O espectro do ruído DM apresenta um decaimento natural de $-40 \mathrm{~dB} / \mathrm{dec}$. A amplitude dos harmônicos é reversamente proporcional ao quadrado da ordem do harmônico, o que quer dizer que a amplitude do harmônico decai com uma taxa de $40 \mathrm{~dB} / \mathrm{dec}$.

A circulação do ruído DM no conversor boost pode ser observado na Figura 6. Nota-se que a circulação do ruído é idêntica a corrente principal do conversor. Da mesma forma, na Figura 7 é apresentada a circulação do ruído para o conversor boost intercalado e na para o conversor dual boost.

A LISN (Line Impedance Stabilization Network), a qual é posicionada na entrada dos conversores, filtra e separa as correntes de ruído, e também proporciona meios para medi-los. A LISN é usada somente durante as medidas de EMI conduzida e fornece uma linha de impedância estabilizada o que garante repetitibilidade dos resultados obtidos, como afirma (Zientarski et al., 2009) e (Mainali and Oruganti, 2010).

Desse modo, o indutor boost é o elemento de maior impacto no ruído conduzido DM já que o mesmo influencia a parte de baixa frequência da norma $(150 \mathrm{kHz}-5 \mathrm{MHz})$ através da amplitude da ondulação de corrente, e também na parte de alta frequência da norma $(5 \mathrm{MHz}-30 \mathrm{MHz})$ através das capacitâncias parasitadas do indutor. Tais capacitâncias proporcionam um caminho de baixa impedância, permitindo a passagem do ruído DM, dependendo da frequência do sinal que percorre o mesmo, (Zientarski et al., 2009). Dessa forma, deve-se estar atento ao projeto do indutor boost, já que o mesmo afeta diretamente na geração da EMI conduzida do tipo DM.

Outro fator que interfere no ruído conduzido DM é a corrente de recuperação reversa do diodo boost. Esta corrente circula pela chave semicondutora durante o turn-off do diodo, inserindo oscilações de alta frequência sobre a corrente do conversor. Tais oscilações geram ruído conduzido DM, como apresentado por (Hertz, 2001). 


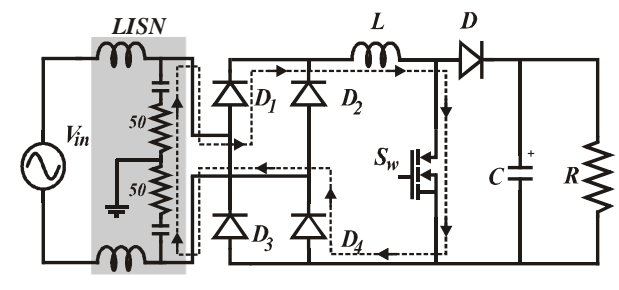

Figura 6: Ruído conduzido DM no conversor boost.

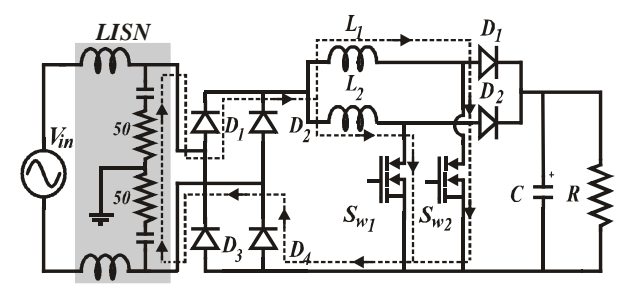

Figura 7: Ruído conduzido DM no conversor boost intercalado.

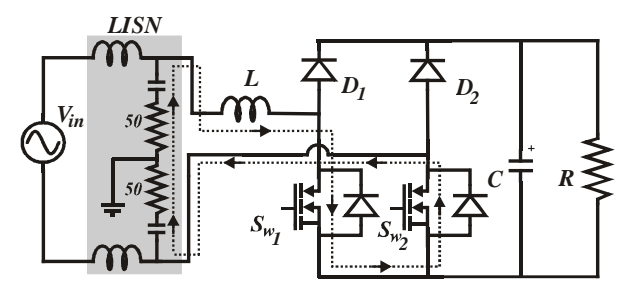

Figura 8: Ruído conduzido DM no conversor dual boost.

\subsection{EMI Conduzida - Modo Comum}

Este tipo de ruído é tipicamente causado pelas altas taxas de variação de tensão e pelas capacitâncias parasitas. No caso do conversor boost PFC, o ruído CM está diretamente relacionado a uma única capacitância parasita, conforme mostraram (Zhang, 1998), (Zhang et al., 2000), (Hertz, 2001) e (Zientarski et al., 2009). Trata-se da capacitância parasita que se estabelece entre a trilha do dreno (no caso do MOSFET) ou no coletor (no caso do IGBT) do interruptor principal e o terra.

Como esse é o nó que apresenta a maior variação de tensão no conversor boost (zero volts quando a chave semicondutora está em condução, e igual a tensão do barramento quando está bloqueada), é nesse ponto que poderá ocorrer a maior fuga de corrente para o terra através de capacitâncias parasitas. A Figura 9 ilustra tal capacitância parasita $\left(C_{p}\right)$, bem como o nó onde ocorre a grande variação de tensão no conversor boost.

Quanto mais rápida a variação de tensão (maior $d v / d t$ ) mais se evidencia o efeito da reatância capacitiva. O resultado é o aparecimento de impulsos (spikes) de corrente no caminho do ruído de modo comum nos momentos em que ocorrem as comutações do interruptor principal. Devido ao fato dessa corrente se dar na forma de impulsos, ela se espalhará no espectro, a partir da frequência de comutação.

A Figura 10 ilustra o caminho percorrido pelo ruído conduzido CM na etapa PFC do conversor boost. Da mesma forma, a Figura 11 apresenta a circulação do ruído para o conversor boost intercalado e, a Figura 12, para conversor dual boost.

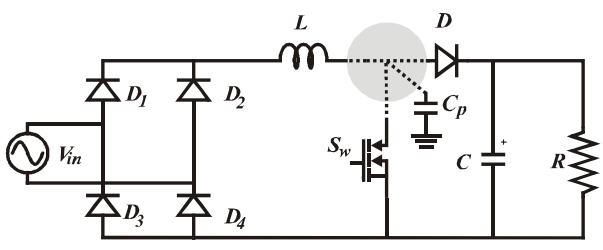

Figura 9: Capacitância parasita no conversor boost.

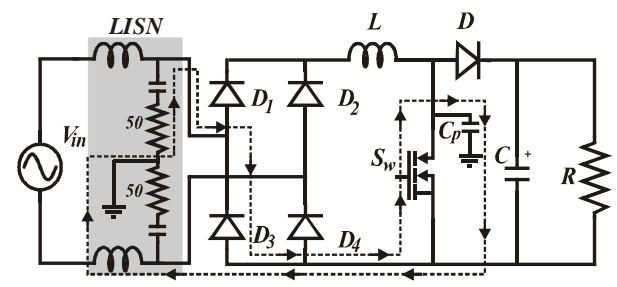

(a) $1^{a}$ etapa de circulação do ruído

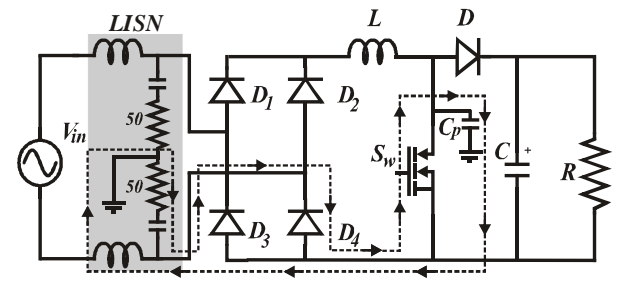

(b) $2^{a}$ etapa de circulação do ruído

Figura 10: Ruído conduzido CM no conversor boost.

Pode ser observado que o ruído CM é ilustrado em duas etapas. Foi apresentado assim, para melhor visualização da circulação do ruído $\mathrm{CM}$, que por definição circula ao mesmo tempo pelo condutor fase e pelo condutor neutro. Logo, a $1^{a}$ etapa de circulação do ruído representa o ruído CM que circula pelo condutor fase, e a $2^{a}$ etapa a circulação pelo condutor neutro. Vale ressaltar ainda que o caminho de circulação do ruído completa seu circuito através das capacitâncias parasitas de toda a montagem. No entanto, como já foi supracitada, a capacitância de maior relevância no conversor boost, é a capacitância que se estabelece entre a trilha do dreno (no caso do MOSFET) ou no coletor (no caso do IGBT) do interruptor principal e o terra, representado nas figuras como capacitor $C_{P}$. 


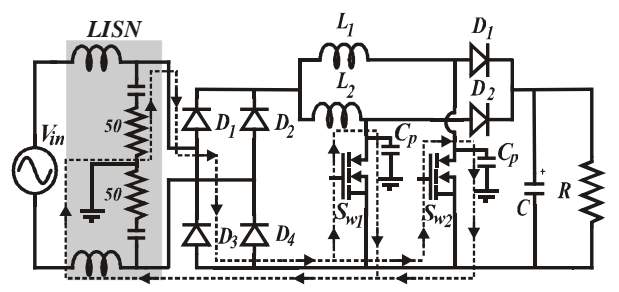

(a) $1^{a}$ etapa de circulação do ruído

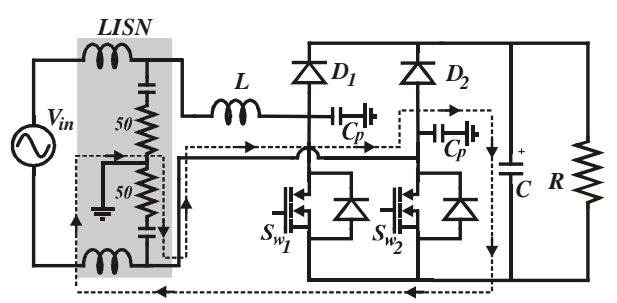

(b) $2^{a}$ etapa de circulação do ruído

Figura 11: Ruído conduzido CM no conversor boost intercalado.

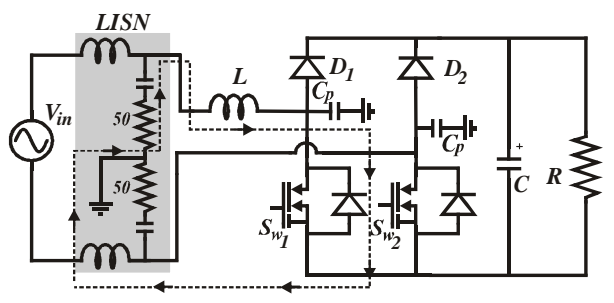

(a) $1^{a}$ etapa de circulação do ruído

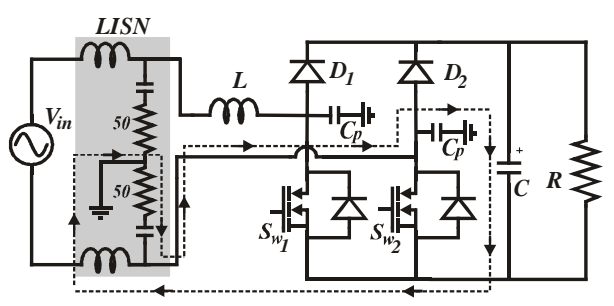

(b) $2^{a}$ etapa de circulação do ruído

Figura 12: Ruído conduzido CM no conversor dual boost.

Pode-se notar ainda que somente na topologia dual boost o ruído circulará pelo indutor boost, o qual esta localizado no lado AC. Este fato contribuirá para atenuação do ruído CM, já que o indutor representará um caminho de alta impedância para a passagem do ruído. Nas topologias boost e boost intercalado isso não ocorre.

Outra característica marcante da topologia dual boost é a presença de uma outra variação de tensão de alta freqüência, o que representa uma fonte pulsante de alta freqüência. Tal fonte de tensão faz com que ruído CM gerado pela topolo- gia dual boost seja maior que dos outros conversores, como apresentado por (Ye et al., 2004) e (Huber et al., 2008).

Para o entendimento de como esta fonte pulsante de alta frequência afeta o ruído $\mathrm{CM}$ deste conversor, há a necessidade de se analisar os modos de operação do mesmo. Pela análise da Figura 13, onde é ilustrado o modo de operação do conversor dual boost, no semi-ciclo positivo, verifica-se que a chave semicondutora $S_{w 1}$ e o diodo $D_{1}$ comutam em alta frequência e o diodo intrínseco da chave semicondutora $S_{w 2}$ é responsável pela retificação. Este diodo da chave semicondutora faz com que, neste semi-ciclo, a diferença de potencial entre o terra de entrada da fonte e o terra de saída do conversor seja igual a zero.

Por outro lado, no semi-ciclo negativo, Figura 14, a chave semicondutora $S_{w 2}$ e o diodo $D_{2}$ comutam em alta frequência e o diodo intrínseco da chave semicondutora $S_{w 1}$ é responsável pela retificação. Analisando a Figura 14 verifica-se que a diferença de potencial entre o terra de entrada da fonte e $o$ terra de saída do conversor é igual a zero quando a chave semicondutora está conduzindo, e igual a tensão do barramento quando a chave semicondutora está bloqueada. Se a mesma análise for realizada para os conversores boost e boost intercalado, é verificado que não há esta diferença de potencial.

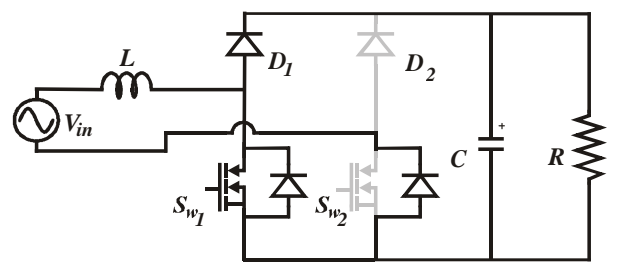

Figura 13: Modo de operação, conversor dual boost, semiciclo positivo.

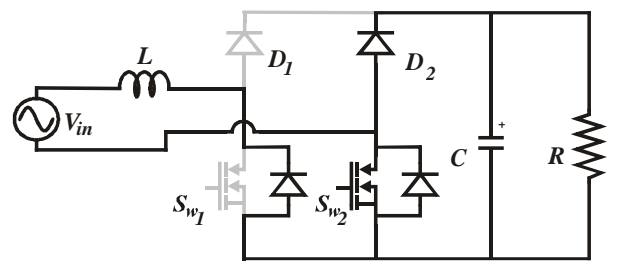

Figura 14: Modo de operação, conversor dual boost, semiciclo negativo.

Esta diferença de potencial representa uma fonte de tensão pulsante de alta frequência, a qual carrega e descarrega todas as capacitâncias parasitas entre o terra de saída e o terra de entrada. Isso resulta em significativo aumento do ruído conduzido CM, fazendo com que este conversor seja o mais ruidoso dos três sob análise. 


\section{CONTROLE DA EMI CONDUZIDA}

O controle da emissão de ruído conduzido se faz necessário sempre que o limite recomendado pela norma for superado. Há basicamente dois métodos de se reduzir o ruído gerado. O primeiro, um método corretivo, o qual faz o bloqueio do ruído existente através de filtragem e segundo, um método preventivo, o qual procura atacar os pontos onde o ruído é gerado ou transmitido para os condutores de entrada. Na maior parte dos casos, ambos os métodos precisam ser utilizados concomitantemente, pois nem sempre é possível um nível de minimização suficiente, através do método preventivo, para se descartar o filtro, conforme (U-Yaisom et al., 2002) e (Pei et al., 2003).

Relativo ao método corretivo, a Figura 15 representa as topologias de filtro mais utilizadas no controle da EMI conduzida. Estes filtros são conhecidos como $\pi$ balanceado e múltiplo $\pi$, de acordo com (Shih et al., 1996), (Pieniz et al., 2006) e (Beltrame et al., 2009). A principal característica desses filtros é a presença de um único indutor acoplado que atua tanto sobre o ruído DM, através da indutância de dispersão das bobinas, como sobre o CM através da impedância do indutor acoplado.

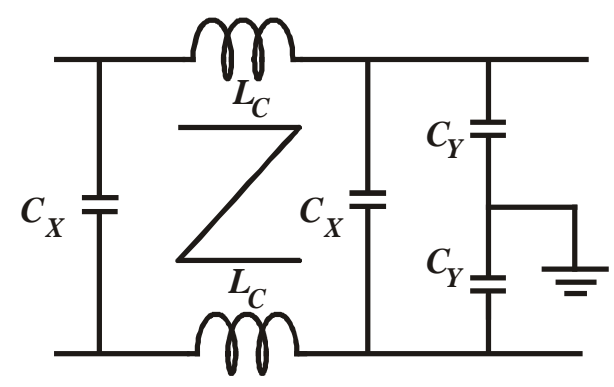

(a)

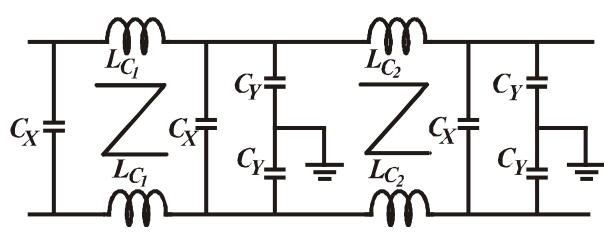

(b)

Figura 15: Filtros de EMI: a) Fitro $\pi$ balanceado, b) Filtro múltiplo $\pi$.

Quanto ao método preventivo de redução do EMI, ou seja, atacar as fontes de ruído ao invés de apenas bloqueá-lo com filtragem, o primeiro passo é o conhecimento da localização das fontes e dos caminhos que o ruído percorre. Como já apresentado anteriormente, o indutor boost tem papel decisivo na redução do ruído DM. Este indutor influencia a parte de baixa frequência da norma $(150 \mathrm{kHz}-5 \mathrm{MHz})$ através da amplitude da ondulação de corrente e também influencia na parte de alta frequência da norma $(5 \mathrm{MHz}-30 \mathrm{MHz})$ através da sua curva de impedância, que pode ser prejudicial devido a presença de parasitas capacitivos no mesmo.

No primeiro caso $(150 \mathrm{kHz}$ a $5 \mathrm{MHz})$, a redução do ruído gerado só é atingida se a indutância do indutor boost for aumentada para diminuir a amplitude da ondulação de corrente (considerando a mesma frequência de comutação). No segundo caso (5 MHz a $30 \mathrm{MHz}$ ), o desafio é a minimização dos parasitas capacitivos do indutor boost, os quais estão ligados à forma como o indutor é confeccionado e aos materiais utilizados. Uma técnica muito utilizada, e apresentada em (Wang et al., 2003), consiste em enrolar os indutores com uma única camada de fio, tanto o do filtro quanto o do conversor boost, pois isso elimina a capacitância que se estabelece entre as camadas. A escolha do material magnético também tem influência na capacitância parasita, pois cada um pode apresentar características elétricas e dielétricas diferentes. Como a primeira camada de espiras fica muito próxima do material magnético, o mesmo pode afetar a capacitância entre espiras consecutivas e consequentemente a capacitância parasita total, conforme mostrado por (Wang et al., 2003).

Quanto ao ruído CM, o objetivo é reduzir as capacitâncias parasitas que se estabelecem entre o nó central da célula de comutação dos conversores, o dissipador e o aterramento. Essa capacitância dependerá da área da trilha do circuito impresso, da área do dissipador e da distância desses elementos até o terminal de aterramento. Logo, para a redução desta capacitância parasita, algumas medidas podem trazer alguns benefícios, como ilustrados em (Rossetto et al., 1998) e (Rossetto et al., 2000):

- Utilizar um material condutor entre o isolante térmico e o dissipador, Figura 16, inserindo dessa forma mais uma capacitância, diminuindo a capacitância total.

- Quando utilizada a isolação, conectar o dissipador ao terminal emissor (IGBT). Essa atitude faz com que o dissipador atue como uma blindagem desviando a corrente para o emissor.

- Ao desenvolver o projeto da placa, tentar deixar o dissipador o mais afastado possível das paredes da caixa do chassi. Além disso, diminuir ao máximo a área da trilha do circuito impresso usada no respectivo nó.

- Reduzir o comprimento dos fios onde há circulação de corrente;

- Entrelaçar, se possível, todas as trilhas críticas da placa de circuito impresso (PCB), e também os fios do enrolamento do indutor, como mostrado na Figura 17. Isto 
mantém as áreas de emissão tão pequenas quanto possíveis, introduzindo também um cancelamento mutuo dos fluxos.

- Redução das áreas dos laços onde há grandes variações de corrente e tensão ( $d i / d t$ e $d v / d t)$, Figura 18. A diminuição das áreas dos laços, através da aproximação das vias de ida e retorno do sinal ajuda a diminuir a possibilidade de emissão e recepção de interferências eletromagnéticas.

Além dos pontos citados anteriormente, ainda existe a opção reduzir a velocidade da comutação, que pode ser controlada facilmente através da resistência de gate, ou seja, da resistência que define a corrente máxima com a qual o terminal de controle da chave semicondutora será carregado. Pode-se diminuir a taxa de variação de tensão $(d v / d t)$ durante as comutações através do aumento da resistência de gate e, como resultado, se diminui a amplitude do ruído de alta frequência gerado conforme Rossetto et al. (1998) e Rossetto et al. (2000). Todavia, essa atitude pode elevar as perdas de comutação, e consequentemente, acarretar em uma degradação do rendimento do conversor.

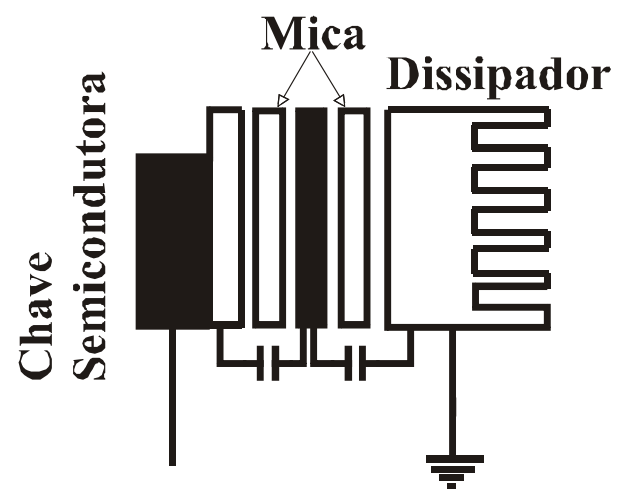

Figura 16: Inserção de capacitância série entre o semicondutor e o dissipador.

\section{PROJETO DO FILTRO DE EMI}

Para o projeto do filtro $\pi$ balanceado ou o múltiplo $\pi$, foram seguidos os passos mostrados por (Pieniz et al., 2006) e (Beltrame et al., 2009). Primeiramente, a corrente de entrada dos conversores foi obtida por simulação. Com esta simulação o ruído conduzido DM pode ser estimado já que o ruído conduzido DM é diretamente dependente da forma de onda da corrente de entrada. Dessa forma, com o ruído DM simulado, a frequência de corte do filtro DM é obtida através da FFT (Fast Fourier Transform) da corrente de entrada. Para definição da freqüência há a necessidade da obtenção do harmônico critico. O harmônico crítico é o primeiro harmô-
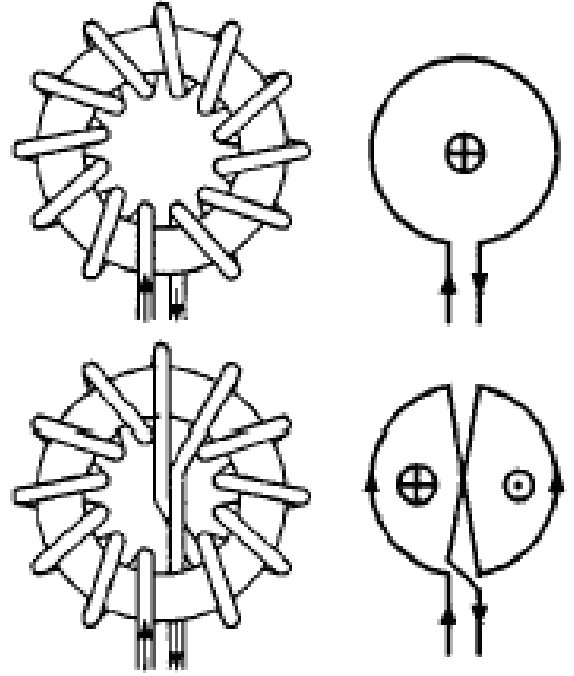

Figura 17: Técnica para redução de EMI nos indutores.

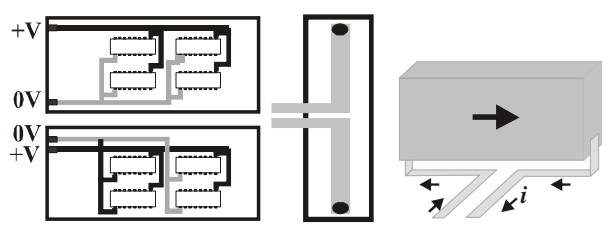

Figura 18: Diminuição das áreas dos laços com aproximação das vias.

nico que entra na faixa da norma de interferência eletromagnética é a frequência de pior caso, ou seja, a necessidade de atenuação máxima para atingir conformidade com a norma. Com esse harmônico critico, traça-se uma reta, com inclinação determinada pela ordem do filtro, a partir do valor de pico do harmônico crítico em direção à origem, até uma linha imaginária paralela ao eixo das frequências, que parte do limite da norma. A intersecção desta reta com a linha imaginária horizontal determina a frequência de corte do filtro DM. A Figura 19 apresenta o harmônico crítico e ilustra como é realizada a obtenção da frequência de corte do filtro. Esta figura, Figura 19, foi obtida através do uso do software MATLAB ${ }^{\circledR}$ apenas para demonstrar o harmônico critico, e a obtenção da frequência de corte.

Com a frequência de corte do ruído DM e com as equações (2) a (5), apresentadas em (Pieniz et al., 2006), determina-se o valor do indutor DM para o filtro $\pi$ ou múltiplo $\pi$.

$$
L_{D M}=\left(\frac{1}{2 \pi f_{c u t}}\right)^{2} \frac{1}{C_{x_{2}}}
$$




$$
\begin{gathered}
L_{D M_{1}}=L_{D M_{2}} \\
L_{C M_{1}}=L_{C M_{2}} \\
C_{X_{1}}=C_{X_{3}}=0.5 C_{X_{2}}
\end{gathered}
$$

Onde:

$L_{D M}$ : Indutância do indutor DM.

$L_{C M}$ : Indutância do indutor CM.

$C_{x}$ - Capacitância do capacitor DM.

$f_{\text {cut }}$ - Frequência de corte do ruído DM.

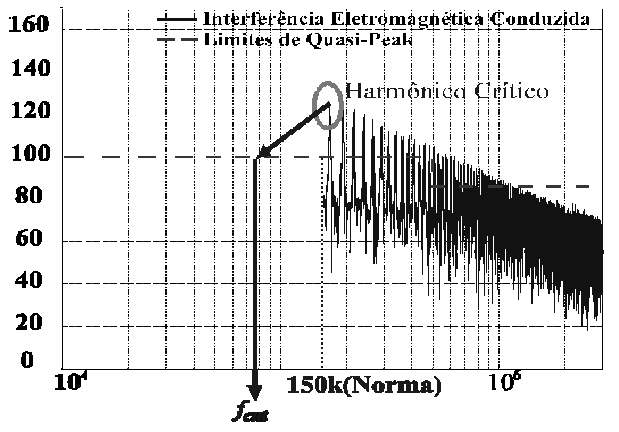

Figura 19: Obtenção da frequência de corte do filtro.

A indutância $L_{D M}$ é indutância de dispersão do indutor acoplado, logo ela se encontra em serie com o mesmo. No entanto, na esta indutância não foi ilustrada na Figura 15. Portanto, tal indutância é obtida da dispersão do indutor $L_{C}$, e é aproximadamente de 0,5 a $2 \%$ da indutância do indutor acoplado, como afirma (Pieniz et al., 2006). Logo, o indutor, $L_{C M}$, é estimado e os capacitores $C_{Y}$ são escolhidos aleatoriamente até atenuação do ruído. Entretanto, os capacitores do filtro precisam ser limitados para não interferir no PFC. Logo, o valor máximo da capacitância equivalente de entrada do filtro é calculado através da equação (6) conforme (Beltrame et al., 2009).

$$
C_{\text {max }}=\frac{P_{\text {min }}}{2 \pi f\left(V_{\text {fase }(r m s)}\right)^{2}} \tan \left(\arccos \left(F P_{\text {min }}\right)\right)
$$

Onde:

$P_{\text {min }}$ - Potência mínima de entrada do conversor.

$$
\begin{aligned}
& F P_{\min } \text { - Fator de potência mínimo desejável. } \\
& f \text { - Frequência de entrada. } \\
& V_{f a s e(r m s)} \text { - Tensão eficaz de entrada. }
\end{aligned}
$$

Entretanto, o uso do filtro de EMI afeta a dinâmica do conversor que pode degradar o desempenho do pré-regulador, pois o filtro afeta a função de transferência do conversor. Como solução, é utilizado um resistor em paralelo com o filtro para amortecer as oscilações causadas pelo filtro de EMI. Contudo, aumenta a potência dissipada o que penaliza sua utilização em diversas aplicações. Uma solução prática é ilustrada na Figura 20, de acordo com (Beltrame et al., 2009). Um capacitor $\left(C_{f}\right)$, é adicionado em série com o resistor $R_{f}$. Dessa forma, nenhuma corrente CC passará pelo resistor $R_{f}$ o que minimiza as perdas. Assim, o cálculo do resistor de amortecimento $\left(R_{f}\right)$ e do capacitor $\left(C_{f}\right)$ é obtido através das equações (7), (8) e (9).

$$
\begin{gathered}
R_{o f}=\sqrt{\frac{L_{c}}{C_{x}}} \\
n=\frac{C_{x}}{C_{f}} \\
R_{f}=R_{o f} \sqrt{\frac{(2+n)(4+3 n)}{2 n^{2}(4+n)}}
\end{gathered}
$$

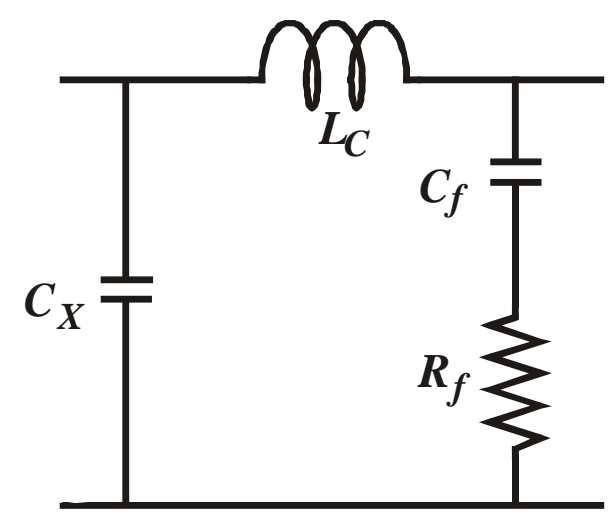

Figura 20: Filtro de entrada amortecido.

\section{RESULTADOS EXPERIMENTAIS}

Nesta seção são apresentados os resultados experimentais para os conversores operando como PFC. A tabela 1 ilustra os parâmetros de projeto dos três conversores utilizado para a obtenção dos resultados experimentais. Deve-se destacar 
que a frequência de comutação do conversor boost intercalado é a metade da frequência dos outros dois conversores. Isso é feito para que a ondulação da corrente de entrada seja a mesma para todos os conversores. Além disso, os valores dos indutores foram calculados de modo que a THD da corrente entrada seja a mesma.

Tabela 1: Parâmetros de Projeto.

\begin{tabular}{|c|c|c|c|}
\hline $\begin{array}{c}\text { Pré- } \\
\text { Reguladores }\end{array}$ & Boost & $\begin{array}{c}\text { Boost In- } \\
\text { tercalado }\end{array}$ & $\begin{array}{c}\text { Dual } \\
\text { Boost }\end{array}$ \\
\hline $\begin{array}{c}\text { Tensão de } \\
\text { entrada }\end{array}$ & $220 \pm 15 \%$ & $220 \pm 15 \%$ & $220 \pm 15 \%$ \\
\hline $\begin{array}{c}\text { Tensão de } \\
\text { saída }\end{array}$ & $400 \mathrm{~V}$ & $400 \mathrm{~V}$ & $400 \mathrm{~V}$ \\
\hline $\begin{array}{c}\text { Potência de } \\
\text { saída }\end{array}$ & $300 \mathrm{~W}$ & $300 \mathrm{~W}$ & $300 \mathrm{~W}$ \\
\hline $\begin{array}{c}\text { Freq. de } \\
\text { comutação } \\
\text { Cap.do } \\
\text { barramento }\end{array}$ & $24 \mathrm{kHz}$ & $12 \mathrm{kHz}$ & $24 \mathrm{kHz}$ \\
\hline Indutor boost & $1,86 m H$ & $1,75 m H$ & $1,45 m H$ \\
\hline
\end{tabular}

Deve-se salientar ainda, que a escolha da freqüência de comutação dos três conversores é obtida conforme apresentado por (Damasceno et al., 2005) e (Sartori et al., 2009). Tais trabalhos apresentam uma metodologia de projeto que define a freqüência de comutação para a obtenção de um ponto de operação ótimo, tendo dessa forma, o menor volume dos magnéticos do indutor boost e dos indutores do filtro de EMI.

A Figura 21(a) apresenta o conversor boost operando como PFC para a carga de $300 \mathrm{~W}$. Da mesma forma, na Figura 21(b) tem-se o conversor boost intercalado, com duas células intercaladas e com uma defasagem de $180^{\circ}$ entre elas. Já na Figura 21(c) tem-se o conversor dual boost para a mesma carga.

O controle dos conversores foi realizado conforme mostrado por (Roggia et al., 2009a; Roggia et al., 2009b). Na tabela 2 é ilustrado o desempenho dos conversores quanto ao fator de potência e THD da corrente de entrada para carga nominal. Todos os conversores apresentaram resultados muito satisfatórios quanto aos dois quesitos analisados.

Tabela 2: Desempenho dos conversores.

\begin{tabular}{|c|c|c|}
\hline Pré-reguladores & FP & THD (\%) \\
\hline Boost & 0,9993 & 4,35 \\
\hline Boost Intercalado & 0,9990 & 4,05 \\
\hline Dual Boost & 0,9897 & 7,98 \\
\hline
\end{tabular}

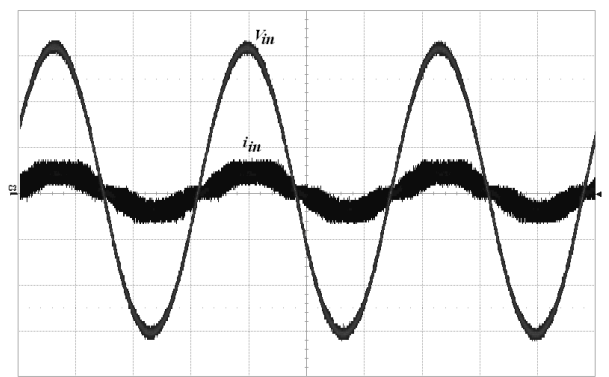

(a) Conversor boost

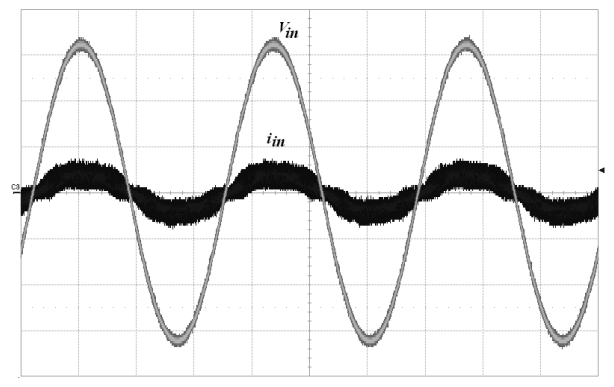

(b) Conversor boost intercalado

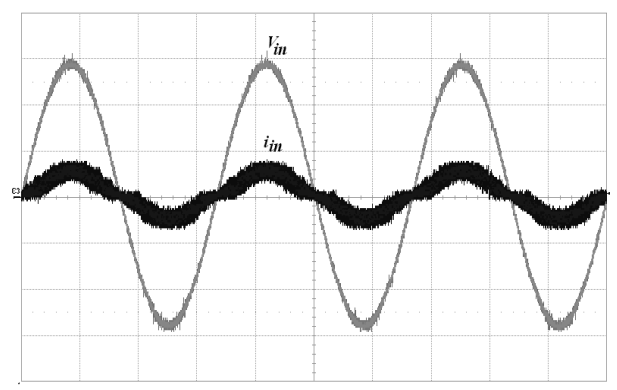

(c) Conversor dual boost

Figura 21: Conversores operando como PFC.

A Figura 22(a) apresenta a medição da EMI conduzida para o conversor boost sem a utilização de filtro. Além disso, podese verificar os limites da norma CISPR 22 de $150 \mathrm{kHz}$ até $30 \mathrm{MHz}$ para valores de quase-pico. Da mesma forma, a Figura 22(b) e a Figura 22(c) apresentam as medições para os conversores boost intercalado e dual boost, respectivamente.

Em cada figura é ilustrado o valor do harmônico crítico e da sua frequência. Com estes valores, o filtro de EMI é projetado conforme mostrado na seção 4. A Figura 23 ilustra o filtro implementado, o qual é um filtro de EMI de $5^{a}$ ordem. $\mathrm{O}$ volume do indutor foi o fator determinante para a escolha da ordem do filtro, pois o filtro de $3^{a}$ ordem resultaria em um indutor muito volumoso.

A Figura 24 apresenta os resultados obtidos com o uso do filtro de EMI projetado. Como pode ser verificado, o filtro 


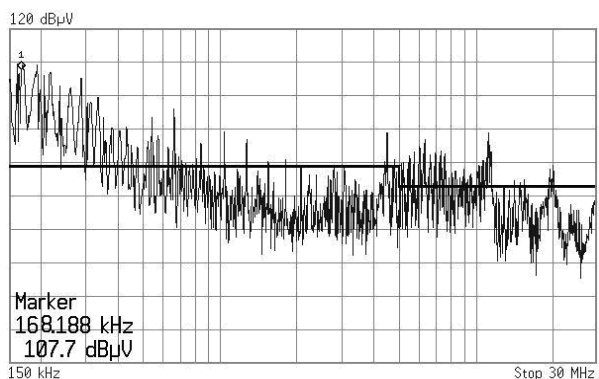

(a) Conversor boost

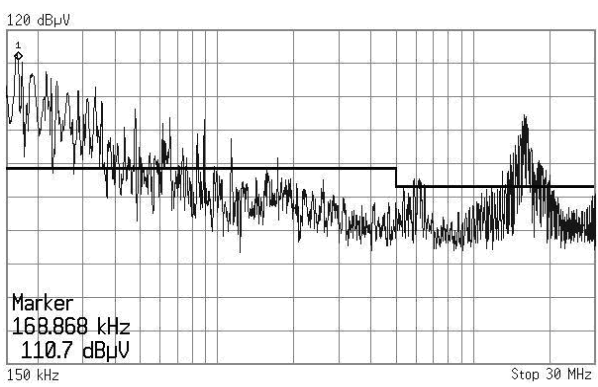

(b) Conversor boost intercalado

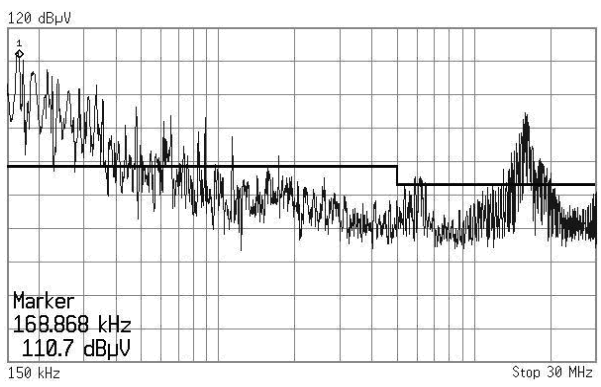

(c) Conversor dual boost

Figura 22: Medição da EMI conduzida sem o uso do filtro.

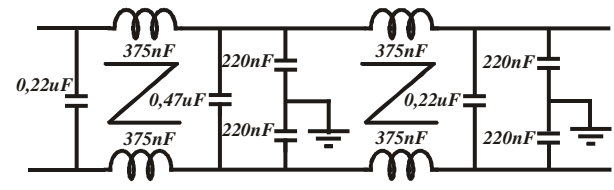

Figura 23: Filtro de EMI utilizado.

de EMI atenuou o ruído para os conversores boost e boost intercalado em quase toda a faixa de frequência. No entanto, perto de $20 \mathrm{MHz}$ existe um pico de interferência fora dos limites da norma. Associa-se este pico às capacitâncias parasitas do indutor boost, a qual está proporcionando um caminho de baixa impedância para a circulação do ruído. Já o conversor dual boost não apresentou o mesmo comportamento dos demais.

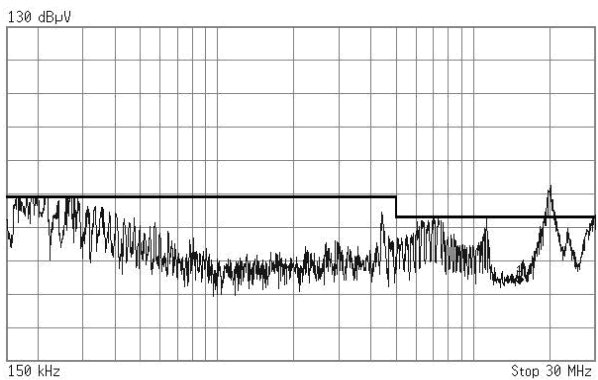

(a) Conversor boost

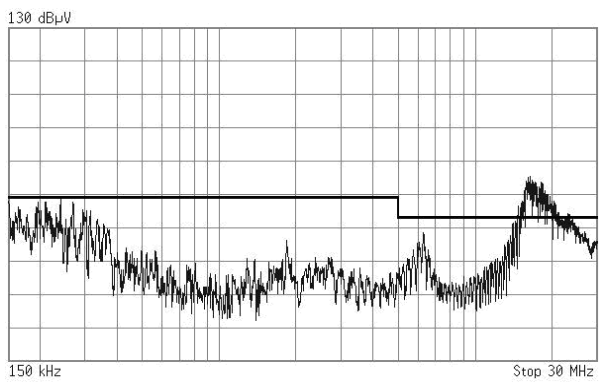

(b) Conversor boost intercalado

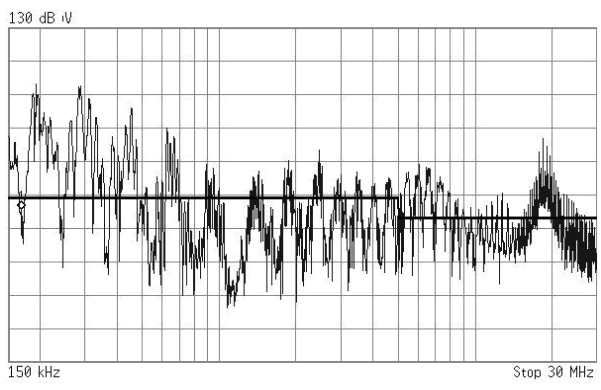

(c) Conversor dual boost

Figura 24: Medição da EMI conduzida com o uso do filtro.

A Figura 25 a mostra a curva de impedância do indutor boost, do conversor boost, na faixa de freqüência de $1 \mathrm{kHz}$ até $30 \mathrm{MHz}$. A obtenção da curva de impedância do indutor foi obitida com a utilização do equipamento Network /Spectrum/Impedance Analyzer modelo 4395A, fabricado pela empresa Agilent Technologies. Através desta curva pode-se ter uma idéia do comportamento da impedância do indutor na faixa de freqüência de EMI.

Observa-se que o comportamento indutivo ocorre até o primeiro pico, quando o mesmo passa a se comportar como um capacitor. A partir desse ponto a impedância passa por sucessivos pontos de picos e vales, sendo que os vales constituem pontos de baixa impedância e facilitam a transmissão do ruído de comutação para os terminais de entrada. Esses vales são determinados pelos parasitas capacitivos do indu- 


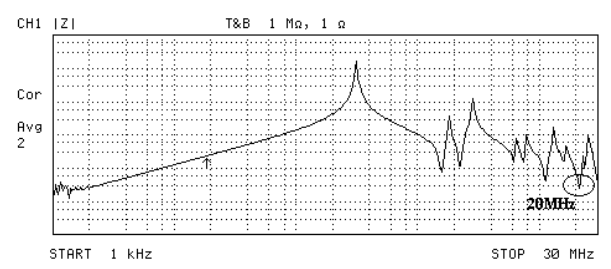

Figura 25: Curva de impedância do indutor do conversor boost.

tor boost. Como resultado, tem-se que o espectro do ruído conduzido DM fica amplificado nas freqüências dos vales.

Aproximadamente em $20 \mathrm{MHz}$, pode ser verificado que há a existência de um vale, que apresenta a menor resistência dos demais. Isso faz com que, nessa frequiência, se tenha um caminho para o ruído conduzido. Além disso, pode-se dizer ainda que esta interferência não é atenuada pelo filtro de EMI devido as não idealidades dos componentes do filtro de EMI, uma vez que os filtros podem possuir zeros, na sua função de transferência, bem abaixo dos $30 \mathrm{MHz}$ que definem o limite superior da norma CISPR 22.

Como alternativa para tentar eliminar o pico de interferência e adequar os conversores as exigências da norma propõe-se a alteração do resistor de gate das chaves semicondutoras, passando de $10 \Omega$ para $100 \Omega$. A alteração dessa resistência resultou na redução de aproximadamente $1 \%$ da eficiência do conversor boost intercalado. Os resultados dessa alteração podem ser vistos na Figura 26(a), 26(b) e 26(c) para os conversores boost, boost intercalado e dual boost. Tal alteração fez com que os conversores boost e boost intercalado se adequaram aos limites da norma, e novamente o conversor dual boost permaneceu com seus limites acima do permitido.

Logo, pode-se afirmar que o conversor dual boost é o que apresenta os maiores níveis de ruído, e associa-se isso a presença da fonte de tensão pulsante já mencionada. Na tentativa de reduzir o efeito desta fonte de tensão pulsante de alta frequência sobre o ruído conduzido CM, utilizou-se a técnica proposta por (Huber et al., 2008). A proposta de Huber é fazer com que a EMI gerada pelo conversor dual seja similar ao conversor boost.

A Figura 27 apresenta a solução utilizada, onde o indutor foi dividido em dois, fazendo com que a amplitude da tensão da fonte pulsante tenha uma redução, já que agora a diferença de potencial entre o terra da fonte e o terra da carga não é mais igual a tensão do barramento, e sim a tensão do barramento menos a queda de tensão sobre o indutor. $\mathrm{O}$ resultado da utilização desta técnica pode ser visto na Figura 28, onde nota-se a redução dos níveis de EMI do conversor e a adequação com a norma. Vale ressaltar que todas as medidas foram obtidas com o equipamento EMC Spectrum Analyzer

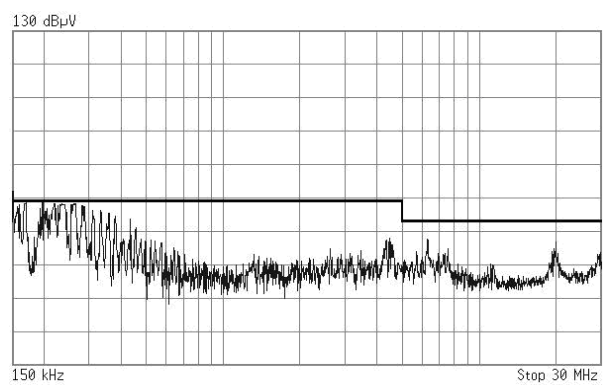

(a) Conversor boost

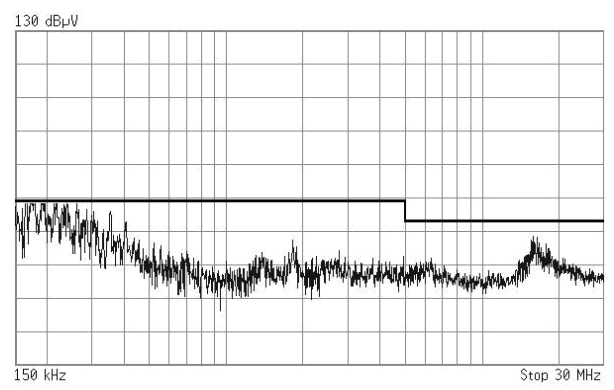

(b) Conversor boost intercalado

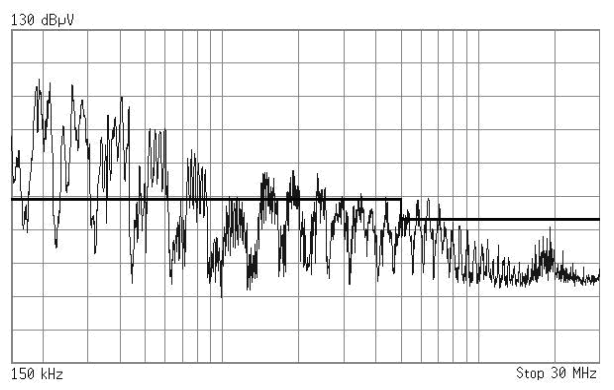

(c) Conversor dual boost

Figura 26: Medição de EMI conduzida com a utilização do filtro e alteração da resistência do gate da chave principal.

and Systems, modelo E7400A, fabricado pela empresa Agilent Technologies.

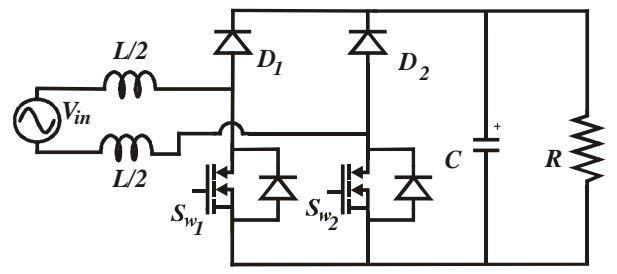

Figura 27: Solução proposta ao conversor dual boost.

Tal alteração, Figura 27, resulta em uma elevação de aproximadamente $53 \%$ nas perdas do indutor boost, já que há um aumento significativo na resistência do enrolamentos. Isto 


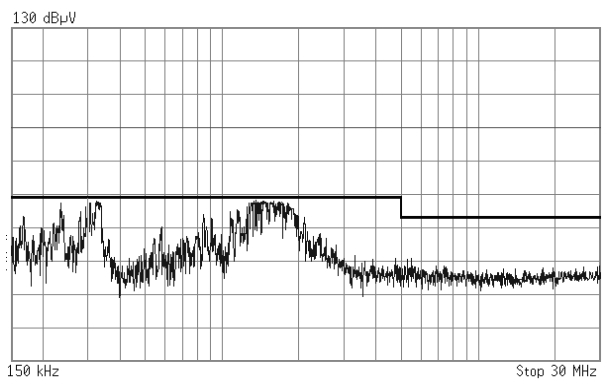

Figura 28: Medição da EMI conduzida do conversor dual boost com alteração proposta e filtro.

ocasiona uma redução de aproximadamente $0,15 \%$ no rendimento total do conversor dual boost.

\section{CONCLUSÃO}

Este artigo apresenta uma investigação da EMI conduzida gerada por conversores boost, boost intercalado com duas células e dual boost operando como PFC para uma carga de $300 \mathrm{~W}$. Foram ilustrados os caminhos percorridos pelos ruídos conduzido do tipo DM e CM nos conversores, bem como o projeto do filtro de EMI.

Resultados experimentais com e sem a utilização do filtro foram apresentados. Conclui-se que, somente a utilização do filtro, não foi suficiente para adequação dos conversores com os limites da norma, especialmente em altas freqüências, devido às capacitâncias parasitas do indutor boost e as não idealidades do filtro de EMI em altas freqüências. Logo, alterou-se a resistência do gate das chaves semicondutoras, o que fez com que os conversores boost e boost intercalado atendessem os limites da norma. Outra alternativa para redução do pico de interferência seria através do projeto do indutor boost com uma única camada. Isso resultaria em uma redução das capacitâncias parasitas do mesmo, mas por outro lado acarretaria no aumento do magnético.

Para o conversor dual boost, os valores de ruído medidos não se enquadraram os limites da norma, mesmo com a utilização do filtro e a alteração do resistor de gate. Dessa forma, solucionou-se esse problema com a divisão do indutor boost em duas partes, fazendo com que a amplitude da fonte de tensão pulsante de alta frequência sofresse uma redução e o mesmo ficou em conformidade com a norma.

No entanto, pode-se afirmar que os três conversores são geradores de EMI conduzida, e que o conversor dual boost é o mais ruidoso das topologias analisadas. Com isso, uma análise detalhada do layout dos conversores, do projeto dos indutores e do filtro de EMI é fundamental para adequar os conversores com os limites da norma CISPR 22.

\section{REFERÊNCIAS}

Beltrame, F., Roggia, L., da Rosa Abaide, A., Schuch, L. and Pinheiro, J. (2009). Emi investigation yield by single-phase pfc pre-regulators, 35th Annual Conference of IEEE Industrial Electronics, 2009. IECON '09., pp. 4128-4133.

Damasceno, D., Schuch, L. and Pinheiro, J. (2005). Design procedure to minimize boost pfc volume concerning the trade-offs among switching frequency, input current ripple and soft-switching, IEEE 36th Power Electronics Specialists Conference, 2005. PESC '05., pp. 23332338.

Hertz, E. (2001). Thermal and emi modeling and analysis of a boost pfc circuit designed using a genetic-based optimization algorithm., Master's thesis, Faculty of the Virginia Polytechnic Institute, Blacksburg, Virginia.

Huber, L., Jang, Y. and Jovanovic, M. (2008). Performance evaluation of bridgeless pfc boost rectifiers, IEEE Transactions on Power Electronics 23(3): 1381-1390.

Lissner, A., Hoene, E., Stube, B. and Guttowski, S. (2007). Predicting the influence of placement of passive components on emi behaviour, European Conference on Power Electronics and Applications, 2007, pp. 1-10.

Lu, B., Dong, W., Wang, S. and Lee, F. (2004). High frequency investigation of single-switch $\mathrm{ccm}$ power factor correction converter, Nineteenth Annual IEEE Applied Power Electronics Conference and Exposition, 2004. APEC '04., Vol. 3, pp. 1481-1487.

Mainali, K. and Oruganti, R. (2010). Conducted emi mitigation techniques for switch-mode power converters: A survey, IEEE Transactions on Power Electronics 25(9): 2344-2356.

Pei, X., Xiong, J., Kang, Y. and Chen, J. (2003). Analysis and suppression of conducted emi emission in pwm inverter, IEEE International Electric Machines and Drives Conference, 2003. IEMDC'03., Vol. 3, pp. 1787-1792.

Pieniz, M., Pinheiro, J. and Hey, H. (2006). An investigation of the boost inductor volume applied to pfc converters, 37th IEEE Power Electronics Specialists Conference, 2006. PESC '06., pp. 1-7.

Roggia, L., Baggio, J. and Pinheiro, J. (2009a). Comparison among digital current controllers applied to power factor correction boost converters, IEEE Energy Conversion Congress and Exposition, 2009. ECCE 2009., pp. 2965-2971. 
Roggia, L., Baggio, J. and Pinheiro, J. (2009b). Predictive current controller for a power factor correction boost converter operating in mixed conduction mode, 13th European Conference on Power Electronics and Applications, 2009. EPE'09., pp. 1-10.

Rossetto, L., Buso, S. and Spiazzi, G. (1998). Conducted emi issues in a boost pfc design, Twentieth International Telecommunications Energy Conference, 1998. INTELEC '98., pp. 188-195.

Rossetto, L., Buso, S. and Spiazzi, G. (2000). Conducted emi issues in a 600-w single-phase boost pfc design, IEEE Transactions on Industry Applications 36(2): 578-585.

Sartori, H., Hey, H. and Pinheiro, J. (2009). An optimum design of pfc boost converters, 13th European Conference on Power Electronics and Applications, 2009. EPE '09., pp. 1-10.

Shih, F.-Y., Chen, D., Wu, Y.-P. and Chen, Y.-T. (1996). A procedure for designing emi filters for ac line applications, IEEE Transactions on Power Electronics 11(1): 170-181.

U-Yaisom, C., Khanngern, W. and Nitta, S. (2002). The study and analysis of the conducted emi suppression on power mosfet using passive snubber circuits, 3rd International Symposium on Electromagnetic Compatibility, 2002, pp. 561-564.

Wang, S., Lee, F. and Odendaal, W. (2003). Single layer iron powder core inductor model and its effect on boost pfc emi noise, IEEE 34th Annual Power Electronics Specialist Conference, 2003. PESC '03., Vol. 2, pp. 847-852.

Yang, L., Lu, B., Dong, W., Lu, Z., Xu, M., Lee, F. and Odendaal, W. (2004). Modeling and characterization of a $1 \mathrm{kw} \mathrm{ccm}$ pfc converter for conducted emi prediction, Nineteenth Annual IEEE Applied Power Electronics Conference and Exposition, 2004. APEC 'O4., Vol. 2, pp. 763-769.

Ye, H., Yang, Z., Dai, J., Yan, C., Xin, X. and Ying, J. (2004). Common mode noise modeling and analysis of dual boost pfc circuit, 26th Annual International Telecommunications Energy Conference, 2004. INTELEC 2004., pp. 575-582.

Zhang, W. (1998). Integrated emi/thermal design for switching power supplies, Master's thesis, Faculty of the Virginia Polytechnic Institute, Blacksburg, Virginia.

Zhang, W., Lee, F. and Chen, D. (2000). Integrated emi/thermal design for switching power supplies, IEEE 31st Annual Power Electronics Specialists Conference, 2000. PESC 00., Vol. 1, pp. 47-52.
Zientarski, J., Pinheiro, J., Hey, H., Beltrame, R. and Candido, D. (2009). A design methodology for boost inductor applied to pfc converters considering the core temperature rise and the conducted emi noise, 13 th $\mathrm{Eu}$ ropean Conference on Power Electronics and Applications, 2009. EPE' '09., pp. $1-10$. 